\title{
Insuficiência renal aguda secundária à sarcoidose
}

\author{
Acute renal failure secondary to sarcoidosis
}

\begin{abstract}
Autores
Marcus Vinícius de Pádua Netto ${ }^{1,2}$

Henrique Vieira de Lima $^{1,2}$

Ana Paula de Souza Borges $^{1}$

Eduardo Moreira dos Santos 3

Émerson Nunes Costa ${ }^{1,2}$

Luiz Cláudio Pádua Netto ${ }^{1}$

'Disciplina de Clínica Médica da Faculdade de Medicina da Universidade Presidente Antônio Carlos (UNIPAC), Araguari

2Departamento de Clínica Médica da Faculdade de Medicina da Universidade Federal de Uberlândia

3Hospital de Clínicas da Faculdade de Medicina da Universidade Federal de Uberlândia
\end{abstract}

Data de submissão: 14/03/2009 Data de aprovação: 24/06/2009

Correspondência para: Marcus Vinícius de Pádua Netto

Rua Marques Póvoa, 513/801

Uberlândia/Minas Gerais

CEP: $38400-438$

Tel: (34) 3236-3079

E-mail:marcus@nanet.com. br/

marcus-netto@uol.com.br

Declaramos a inexistência de conflitos de interesse.

\section{Resumo}

A sarcoidose é uma doença sistêmica de etiologia desconhecida, caracterizada pela inflamação crônica granulomatosa, que acomete com maior frequência os pulmões, a pele e os olhos e, muito raramente, detectamos envolvimento renal na patologia. Relatamos aqui o caso de uma paciente de 47 anos, branca, internada em uma unidade de emergência de Hospital Universitário, devido a sintomas e achados radiológicos sugestivos de pneumonia. Desde o início da internação ela se apresentava com quadro laboratorial de insuficiência renal aguda, inicialmente atribuída ao histórico recente de uso abusivo de anti-inflamatórios. No entanto, devido à evolução arrastada e aparentemente desfavorável, inclusive com necessidade de terapia renal substitutiva (TRS), a paciente foi submetida a uma biópsia renal para esclarecimento diagnóstico e avaliação prognóstica. A descrição histológica mostrava um quadro de nefrite intersticial aguda granulomatosa, característico de sarcoidose renal, diagnóstico confirmado após revisão do prontuário médico, que revelava internação anterior devido a evento pulmonar da doença. Iniciado tratamento com prednisona $1 \mathrm{mg} / \mathrm{kg} / \mathrm{dia}$, a paciente evoluiu com melhora do quadro de insuficiência renal aguda, não mais necessitando de TRS. Atualmente, a paciente é mantida em acompanhamento ambulatorial com função renal estável.

Palavras-chave: sarcoidose, insuficiência renal aguda, nefrite intersticial aguda, hipercalcemia.

[J Bras Nefrol 2009;31(3):223-227]@Elsevier Editora Ltda.

\section{Abstract}

Sarcoidosis is a systemic disease of unknown etiology, characterized by chronic granulomatous inflammation, which affects most frequently the lungs, skin, and eyes, and most rarely the kidneys. We report the case of a 47-year-old, white, woman admitted to the emergency unit of a university-affiliated hospital due to symptoms and radiologic findings suggestive of pneumonia. Since the beginning, her laboratory tests showed acute renal failure, initially attributed to the recent history of abuse of anti-inflammatory drugs. However, because of her protracted and apparently unfavorable evolution, requiring renal replacement therapy (RRT), she underwent renal biopsy for diagnosis and prognostic assessment. Histology showed acute granulomatous interstitial nephritis, characteristic of renal sarcoidosis, which was confirmed after review of the medical record disclosing a previous admission due to lung involvement of the disease. Treatment with prednisone $(1 \mathrm{mg} /$ $\mathrm{kg} /$ day) was initiated, acute renal failure improved, and the patient no longer required RRT. Currently she is followed up on an outpatient service, and her renal function is stable.

Keywords: sarcoidosis, acute renal failure, acute interstitial nephritis, hypercalcemia. 


\section{INTRODUÇÃO}

A sarcoidose é uma doença inflamatória crônica granulomatosa multissistêmica que acomete com maior frequência pessoas jovens e tem como manifestações clínicas mais comuns acometimento torácico (ganglionar e parênquimatoso pulmonar), cutâneo e ocular. Embora de apresentação clínica facilmente detectável, o envolvimento renal é uma forma rara da patologia, encontrado em 20 a $30 \%$ das necropsias realizadas em pacientes portadores de sarcoidose. ${ }^{1}$

As formas mais comuns de apresentação da sarcoidose renal estão relacionadas às alterações no metabolismo do cálcio, a saber: hipercalcemia e hipercalciúria, uropatia obstrutiva, doenças tubulointersticiais e doenças glomerulares. ${ }^{2}$

A hipercalciúria é a grande responsável por alterações agudas da função renal, seja por provocar defeitos na concentração urinária, nefrocalcinose, acidose tubular renal ou litíase renal, fato que dificulta bastante o diagnóstico de envolvimento renal na sarcoidose.

Os quadros de hipercalcemia prolongada que se desenvolvem na sarcoidose induzem vasoconstrição intrarrenal, que, com a evolução da doença e o retardo no diagnóstico e tratamento, acarreta isquemia persistente, podendo, dessa forma, levar à diminuição da filtração glomerular e à necrose tubular aguda isquêmica - que, dependendo da gravidade, pode retardar a recuperação ou evoluir com perda variável da função renal. ${ }^{2}$

Diante disso, a apresentação desse caso tem como objetivo principal, além de descrever o primeiro caso de acometimento renal em sarcoidose na instituição, estimular o estudo sistemático da função renal em pacientes portadores dessa doença e alertar para sua investigação nos pacientes com insuficiência renal aguda de evolução desfavorável, principalmente quando associados à hipercalcemia.

\section{Relato de Caso}

Paciente sexo feminino, branca, 47 anos, hipertensa de longa data, em uso irregular de medicação anti- hipertensiva, procurou serviço de pronto atendimento no dia 3/12/2007, com queixa de tosse produtiva, febre e dispneia de início recente. Ao exame físico, apresentava crepitação principalmente em bases pulmonares bilateralmente, febre de $38,7^{\circ} \mathrm{C}$, edema acentuado e depressivo de membros inferiores e nível pressórico de 164 x 118 mmHg.

A paciente referia ainda que, para alívio de dor dentária, estava em uso de anti-inflamatório não esteroidal há cerca de um mês, sem receita médica, e em uso recente de formulação de cálcio para tratamento de osteoporose (SIC).

Solicitada radiografia de tórax para avaliação do quadro pulmonar, diagnosticou-se quadro de pneumonia, com a prescrição de levofloxacim $500 \mathrm{mg} / \mathrm{dia}$ endovenoso, além de instituição de tratamento anti-hipertensivo.

Concomitante à internação e ao início do tratamento, também foram solicitados exames de bioquímica, que evidenciaram elevação dos níveis de ureia, creatinina e cálcio iônico (Tabela 1).

Internada no Setor de Nefrologia para propedêutica adequada da insuficiência renal aguda, que tinha como principal hipótese diagnóstica necrose tubular aguda, provavelmente secundária ao uso abusivo de anti-inflamatório, e início de TRS para tratamento do quadro de hipervolemia, síndrome urêmica, hipertensão de difícil controle, bem como para tratamento da hipercalcemia apresentada, o que, diante da hipervolemia apresentada, impossibilitou tentativa de tratamento clínico por meio de hiper-hidratação com SF $0,9 \%$ associado a furosemida.

A ultrassonografia renal mostrou nefropatia difusa à esquerda, com manutenção da diferenciação córticomedular, o que sugeria processo renal agudo acometendo a paciente. Após aproximadamente quatro semanas de internação, devido à evolução não esperada para o quadro, com manutenção de níveis elevados das escórias nitrogenadas apesar da melhora da diurese e controle da pressão arterial, optou-se pela realização de biópsia renal para melhor avaliação diagnóstica e prognóstica do quadro de insuficiência renal. 
À biopsia renal, visualizou-se fragmento totalmente constituído por granuloma de células epitelioides, reticulogênico, com eventuais áreas de necrose central de aspecto fibrinoide. Infiltração linfocitária estava presente entre os granulomas (Figuras 1A e 1B) e a pesquisa de bacilo álcool-ácido resistente (BAAR) e de fungos foi negativa.

$\mathrm{Na}$ avaliação do histórico médico da paciente constava, há três anos, investigação de adenomegalia cervical generalizada por meio de biopsia de um dos linfonodos, cujo laudo do exame anatomopatológico descrevia uma inflamação granulomatosa tipo sarcoide, com grande quantidade de granulomas de células epitelioides multinucleadas, tipo Langerhans, sem necrose caseosa, com pesquisa de BAAR e de fungos negativa, tendo sido estabelecido o diagnóstico de sarcoidose, com a prescrição de prednisona $60 \mathrm{mg}$ por dia. A paciente, porém, por conta própria, suspendeu o uso do medicamento após oito meses de tratamento, perdendo o seguimento no serviço.

A partir da descrição anatomopatológica do fragmento renal, da pesquisa negativa de BAAR e de fungos e do diagnóstico prévio de sarcoidose relatado no prontuário médico, considerou-se o quadro de sarcoidose renal a causa da insuficiência renal aguda apresentada pela paciente.

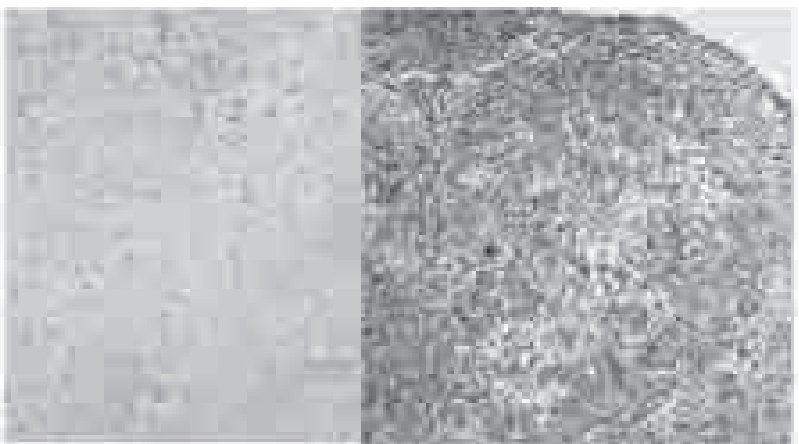

Figura 1A.

Fragmento renal (HE) (reticulina).
Instituiu-se, como tratamento inicial, prednisona, na dose de $40 \mathrm{mg} / \mathrm{dia}$, tendo a paciente recebido alta hospitalar em 16/01/2008, já com melhora da função renal, tendo sido suspensa TRS após seis sessões. Atualmente, é mantida em seguimento ambulatorial. Desde então, apresenta função renal estável (Tabela 2).

\section{Dıscussão}

A insuficiência renal aguda (IRA) ocorre em cerca de 2 a $5 \%$ dos pacientes hospitalizados, atingindo taxas de mortalidade de até $62 \%$, dependendo da presença de comorbidades e de suas complicações. ${ }^{1}$ Qualquer que seja o mecanismo que leve à IRA, pode-se verificar incapacidade de manter balanço hídrico, acúmulo de minerais e metabólitos e sintomas de uremia, o que, em alguns casos, demanda terapia renal substitutiva (TRS), a qual eleva ainda mais os índices de morbidade e mortalidade relacionados à IRA. ${ }^{1}$

Doença de causa desconhecida, a sarcoidose recebeu esse nome devido às características das lesões encontradas, que se assemelham ao sarcoma. ${ }^{2,3} \mathrm{~A} \mathrm{pa-}$ tologia afeta adultos jovens de ambos os sexos, envolvendo primariamente o sistema reticuloendotelial, mas afeta todos os tecidos e órgãos do corpo, preferencialmente o parênquima pulmonar e os linfonodos pulmonares peri-hilares. ${ }^{3}$ Ocasionalmente há comprometimento extrapulmonar, e o sistema nervoso, o trato gastrointestinal, a pele e o fígado são afetados em cerca de $10 \%$ dos casos. ${ }^{3,4}$

Clinicamente importante, o envolvimento renal somente é um problema ocasional na sarcoidose, podendo ocorrer como consequência de hipercalcemia, hipercalciúria, doença glomerular ou nefrite intersticial granulomatosa. Nos casos de sarcoidose, o desenvolvimento de insuficiência renal aguda é pouco comum e verificado em apenas $1 \%$ dos portadores da doença. A evolução para doença renal crônica é raríssima e ocorre principalmente nos pacientes que desenvolvem nefrocalcinose severa. ${ }^{2,4,5}$

Tabela 2 FUnçĀO RENAL EM 04/09/08, SEGUIMENTO AMBULATORIAL

\begin{tabular}{|ccc|} 
& Valor & Valor de Referência \\
\hline Ureia & 90 & $16,7-45,9 \mathrm{mg} / \mathrm{dL}$ \\
\hline Creatinina & 2,0 & $0,6-1,0 \mathrm{mg} / \mathrm{dL}$ \\
\hline Proteinúria/24h & 648 & $24-141 \mathrm{mg} / 24 \mathrm{~h}$ \\
\hline Clearance de creatinina & 58,4 & $75-115 \mathrm{mg} / \mathrm{mim}$ \\
\hline Cálcio & 5,1 & $4,4-5,4 \mathrm{mg} / \mathrm{dL}$ \\
\hline
\end{tabular}


A hipercalcemia é achado comum na sarcoidose, chegando a ser encontrada em 10 a $20 \%$ dos pacientes. Decorre do aumento da absorção intestinal de cálcio e da reabsorção óssea de cálcio, secundárias à elevação dos níveis da vitamina $\mathrm{D}$ ativa (calcitriol) produzidos por macrófagos ativados presentes nos granulomas, e é responsável por algumas das manifestações renais desencadeadas pela patologia. ${ }^{6,7}$

Nos pacientes com quadro de hipercalcemia, a piora da função renal é justificada pela intensa vasoconstrição resultante, o que leva à diminuição do ritmo de filtração glomerular e à necrose tubular aguda isquêmica. ${ }^{6}$

Outro achado comum na sarcoidose, a hipercalciúria pode estar presente em metade dos casos e levar à insuficiência renal aguda por meio de alterações do mecanismo de concentração urinária, nefrocalcinose ou nefrolitíase, que chega a acometer mais de $15 \%$ dos pacientes. ${ }^{2,7-10}$

Embora seja raro e de pouco entendimento o verdadeiro mecanismo de desenvolvimento da lesão, relatos de casos de acometimento glomerular na sarcoidose têm sido feitos e tipos histológicos variados, como glomerulonefrites por lesão mínima, glomeruloesclerose segmentar e focal, nefropatia membranosa, glomerulonefrite membranoproliferatia, nefropatia por IgA e glomerulonefrite crescêntica, já foram descritos. ${ }^{8}$

Aproximadamente $20 \%$ dos pacientes com sarcoidose apresentam inflamação granulomatosa nos rins. A nefrite intersticial granulomatosa comum na sarcoidose foi descrita pela primeira vez em 1933, com prevalência estimada em séries post-mortem que variam de $7 \%$ a $27 \% .6,9,10$

Entretanto, o desenvolvimento de manifestações clínicas e laboratoriais de insuficiência renal não é comum nesses casos de nefrite intersticial granulomatosa e, quando presente, é de intensidade leve a moderada. Embora existam relatos de rápida e progressiva queda da função renal em pacientes com sarcoidose, na maioria dos casos o diagnóstico é difícil, devido à presença concomitante de nefrocalcinose. ${ }^{10-13}$

Em uma série relatada por Brause et al., a causa da insuficiência renal por sarcoidose em cinco de seis pacientes foi nefrite intersticial granulomatosa não caseosa. Dois desses pacientes, já sabidamente portadores da doença, apresentavam hipercalcemia antes do comprometimento renal. Nefrocalcinose hipercalcêmica provocou lesão renal no outro paciente dessa série. ${ }^{11}$

No caso relatado, de uma paciente com história prévia de sarcoidose, foram encontradas na biópsia renal áreas de infiltrado inflamatório entre os granulomas, o que define um quadro de nefrite interstical secundária à sarcoidose, porém com rápido e grave comprometimento da função renal, expresso tanto clínica quanto laboratorialmente com retenção hídrica, hipertensão e síndrome urêmica, demandando, portanto, início de TRS.

O diagnóstico diferencial dos casos de nefrite intersticial é amplo. A maioria está relacionada a reações de hipersensibilidade induzidas por drogas, à própria sarcoidose, a infecções, outras doenças granulomatosas e inflamatórias sistêmicas. ${ }^{14-16}$

Ponce et al. relataram um caso de insuficiência renal aguda cuja etiologia aventada, a princípio, era o abuso de AINE. No entanto, como havia hipercalcemia, tal hipótese foi afastada. $\mathrm{O}$ achado de linfadenopatia mesentérica à TC, a recorrência de insuficiência renal e da hipercalcemia com a suspensão da corticoterapia e a elevação dos níveis da ECA plasmática apoiavam o diagnóstico de sarcoidose. $^{6}$

No caso aqui descrito, diante do histórico recente de uso de AINE, inicialmente atribuíram-se como causas o fator etiológico da insuficiência renal aguda e a hipercalcemia ao uso de formulações contendo cálcio para o tratamento de osteoporose citado pela paciente, o que, de pronto, dificultou que se aventassem outras possibilidades diagnósticas.

Os pacientes portadores de sarcoidose, de modo geral, respondem muito bem à terapia com corticosteroides por via oral mesmo em baixas doses (20 a 40 $\mathrm{mg} / \mathrm{dia})$, que deve ser administrado por um período de 8 a 12 semanas, com posterior redução lenta e progressiva até atingir 10 a $20 \mathrm{mg} / \mathrm{dia}$, e então mantidos assim por até 12 meses. Nos casos de sarcoidose renal, doses ainda menores, 10 a $20 \mathrm{mg} /$ dia de 8 a 12 semanas, são suficientes para o tratamento adequado do quadro. ${ }^{15-19}$

Para os casos resistentes, que apresentam recidivas frequentes ou naqueles pacientes que não toleram uso prolongado de corticosteroides, os pacientes podem ser tratados com ciclofosfamida, infliximabe, azatioprina e, mais recentemente, micofenolatomofetil com vários graus de resposta. ${ }^{19}$

Em nosso caso, inicialmente estabeleceu-se a dose protocolar de $40 \mathrm{mg}$ e, embora o tempo de tratamento recomendado na literatura seja menor, optamos por manter o tratamento prolongado ( 3 meses) diante da inicial melhora com interrupção do tratamento dialítico e redução lenta mas progressiva dos níveis de creatinina.

Dados da literatura sugerem a prevenção de progressão da insuficiência renal ulterior à manifestação aguda da sarcoidose com a utilização de corticoterapia. ${ }^{19}$ 


\section{ConcLusão}

O acometimento renal é uma forma rara de apresentação da sarcoidose, muitas vezes não diagnosticada. No entanto, a sarcoidose deve ser sempre lembrada como diagnóstico diferencial dos quadros de insuficiência renal aguda de evolução desfavorável, mesmo na ausência de manifestações pulmonares ou cutâneas; até porque a resposta ao tratamento com corticosteroides é quase sempre observada, favorecendo a reversão do quadro, evitando perda progressiva e definitiva da função renal. Vale ressaltar que muitas vezes o diagnóstico dessa condição pode ser firmado apenas por meio da biópsia renal, em face das poucas manifestações clínicas encontradas nesses pacientes.

\section{Referências}

1. RL Metha, B McDonald, FB Gabbai. Acute Renal Failure in Medical and Surgical Intensive Units- a One Year Prospective Study. Ren Fail 2003; 60:1154-63.

2. Dahl K, Canetta PA, D’Agati VD, Radhakrishnan J. A 56-year-old Woman with Sarcoidosis and Acute Renal Failure. Kid Int 2008; 74:817-21.

3. Studdy PR, Bird R, Neville E, James DV. Biochemical findings in sarcoidosis. J Clin Pathol 1980; 33:528-33.

4. Newman LS, Rose CS, Maier LA. Sarcoidosis. N Engl J Med 1997; 336:1224-34.

5. Sheffield EA. Pathology of Sarcoidosis. Clin Chest Med 1997; 18:741-53.

6. Murther RS, McCarron DA, Bennett WM. Renal Manifestations of Sarcoidosis. Arch Intern Med 1981; 141:643-5.

7. Rizzato G, Colombo P. Nephrolithiasis as a presenting feature of chronic sarcoidosis: A prospective study. Sarcoidosis Vasc Difuse Lung Dis 1996; 13:167-72.
8. Appel GB, Radhakrishnan J, D’Agati V. Secondary Glomerular Diseases. In: Brenner BM, Rector FC (eds.). Brenner \& Rector's The Kidney. 7 ed. Philadelphia: Saunders, 2004, pp. 1382-482.

9. Woywodt A, Schneider W, Goebel U, Luft FC. Hypercalcemia due to tale granulomatosis. Chest 2000; 117:1195-6.

10. Sharma, Om P. Hypercalcemia in Granulomatous Disorders: a Clinical Review. Curr Opi Pulm Med 2000; 6:442-7.

11. M Brause, K Magnunsson, S Degenhardt, V Helmchen, B Grabensee. Renal Involvement in Sarcoidosis - a Report of 6 cases. Clin Neprhol 2002: 57:142-8.

12. Cruzado JM, Poveda R, Mana J et al. Interstitial Nephritis in Sarcoidosis: Simultaneus Multiorgan Involvement. Am J Kidney Dis 1995; 26:947-51.

13. Berner B, Shulz E, Wieneke U, Reuss-Borst MA, Sattler B, Muller GA. Rasch-Progrediente Niereninsuffizienz als Primar-Manifestation Einer Systemischen Sarkoidose. Med Klin 1999; 94:990-4.

14. Branson JH, Park JH. Sarcoidosis Hepatic Involvement: Presentation of a Patient with Fatal Liver Involvement Including Autopsy Findings and Review of Evidence of Sarcoid Involvement of Liver as Found in Literature. Ann Intern Med 1954; 40:111-4.

15. FJ Casella M Allan. The Kidney in Sarcoidosis. J Am Soc Nephrol 1993; 3:1555-62.

16. G Rizzato. Extrapulmonary Presentation of Sarcoidosis. Curr Opi Pulm Med 2001; 7:295-7.

17. T McCurley, J Salter, A Glick. Renal Insufficiency in Sarcoidosis. Arch Pathol Lab Med 1990; 114:488-92.

18. Sitirling RG, Culliman P, Des Bois R. Sarcoidosis. In: Schwarz MI, King Jr TE (eds.). Interstitial Lung Diseases. BC Decksdon 1998; 279-323.

19. Rajakariar R, Sharples EJ, Raftery MJ, Sheaff M, Yaqoob MM. Sarcoid Tubulo-Interstitial Nephritis: LongTerm Outcome and Response to Corticosteroid Therapy. Kid Int 2006; 70:165-9. 